Original Research Paper

\title{
Acetylation Process and Antioxidant Activity of a Polysaccharide from Loquat (Eriobotrya japonica) Leaves
}

\author{
Yiting Xue, Xiao Ding, Ze Zhang, Xinyi Wu, \\ Xiaolong Cao, Weizhi Zhang, Jiehan Zhang and Yiyong Chen* \\ School of Biology and Food Engineering, Changshu Institute of Technology, Changshu 215500, China
}

\author{
Article history \\ Received: 20-10-2020 \\ Revised: $18-12-2020$ \\ Accepted: $31-12-2020$ \\ Corresponding Author: \\ Yiyong Chen \\ School of Biology and Food \\ Engineering, Changshu \\ Institute of Technology, \\ Changshu 215500, China \\ Email: greenpop6688@126.com
}

\begin{abstract}
Chemical modifications of polysaccharides can provide an opportunity to obtain new agents with possible therapeutic uses. In this study, a water-soluble polysaccharide extracted from Loquat Leaves (coded LLPs) was purified by the column chromatography, which was used to investigate its acetylation process by the acetic anhydride method. The Degree of Substitution (DS) was used as the index, the effect of acetylated reagent amounts, reaction temperature and reaction time on DS was investigated. Acetylation process of LLPs was optimized by Response Surface Methodology (RSM) coupled with Box-Behnken design based on the results of single factor experiments. Characterization and antioxidant activities of Acetylated LLPs (Ac-LLPs) were investigated. Results showed that the optimal acetylation process of LLPs was obtained as following: Acetylated reagent amounts $2.60 \mathrm{~mL}$, reaction time $2.10 \mathrm{~h}$, reaction temperature $31^{\circ} \mathrm{C}$. Under the optimal acetylation process, the maximum DS was 0.3570 . Moreover, the analysis of Fourier-Transform Infrared spectroscopy (FT-IR) confirmed that the structural modification of AcLLPs was successful. In addition, the antioxidant activities assay in vitro suggested that Ac-LLPs possessed better DPPH radical scavenging capacity and stronger hydroxyl radical scavenging capacity compared with LLPs, whereas the scavenging capacity of LLPs on superoxide anion radical was slightly higher than Ac-LLPs. The results indicated that Ac-LLPs had enormous potential to become a new food additive or antioxidant supplement in food and pharmaceutical industries.
\end{abstract}

Keywords: Polysaccharides from Loquat Leaf (LLPs), Acetylation, Response Surface Methodology (RSM), Antioxidant Activity

\section{Introduction}

Reactive Oxygen Species (ROS) are generated in normal metabolic process or derived from exogenous pollutants (Papas, 1999). A number of evidences have shown that continuously producing ROS can attribute to various diseases, including cancers, diabetes, hyperlipidemia, liver cirrhosis, cardiovascular diseases (Valko et al., 2007; Yang et al., 2013). It is believed that antioxidant supplements of foods are helpful for reducing oxidative damages to human. So, there is a strong need for new antioxidants in searching and identification.

Plants are important sources of natural antioxidants (Lae et al., 2019; Zare, 2019; Beridze et al., 2020). In recent years, some natural polysaccharides have been proved to be a class of good free radical scavengers, which can remove the harmful ROS (e.g., superoxide anion and hydroxyl radical) in cells to treat or prevent the body disorders (Ananthi et al., 2010; Wang et al., 2009). It has been proved that introducing other groups into polysaccharides, such as acetylation, carboxymethylation, sulfation, phosphorylation (Liu et al., 2012; Ma et al., 2012), can not only change physicochemical properties and enhance biological activities, but also generate new activities (Li et al., 2016; Lu et al., 2012). Du et al. (2014) found that acetylated tremella polysaccharide produced a new immunomodulatory activity. Xie et al. (2015) indicated that acetylation could significantly increase the antioxidant activity of polysaccharides in leaves of $C$. chinensis, but the monosaccharide composition of the polysaccharides did not change. Chen et al. (2014) confirmed that acetylated polysaccharide of Ganoderma lucidum had stronger antioxidant ability with appropriate 
Degree of Substitution (DS) and low molecular weight. Song et al. (2013) found Pumpkin polysaccharide modified by acetylation was found to significantly enhance antioxidant activity, which positively correlated with the DS of acetyl group. These studies suggested that acetylated polysaccharides is expected to become a new material of food and pharmaceutical industries, including nutriment, food additive, or antioxidant supplement.

Loquat (Eriobotrya japonica L.), a fruit tree and a well-known medical plant belonging to the Rosaceae family, is widely distributed in Southeast Asia (Liu et al., 2016). In China, loquat leaves are usually used as a traditional Chinese medical or a raw material of tea on account of the therapeutic effect on cough, inflammation, nausea, chronic bronchitis (Zar et al., 2014; Chen et al., 2017). Polysaccharides are the major bioactive ingredients of loquat leaves, which take up about $3.62 \sim 5.29 \%$ of loquat leaves with obvious antioxidant, anti-tumor, anti-inflammatory, anti-obesity and antifatigue effects (Wu et al., 2018; Shih et al., 2010; Cha et al., 2011). As the structure characterization technology of polysaccharides becomes more and more reliable (Agsalda-Garcia et al., 2020; Trang et al., 2020), polysaccharides extracted from Loquat Leaves (LLPs) have potential application in the functional food and pharmaceutical industries. However, whether the antioxidant activity of LLPs is influenced by chemical modification is still unknown.

For further researching and utilizing LLPs, a novel LLPs was extracted and purified by DEAE-Sepharose CL-6B and Sephadex G-200 column chromatography. DS was used as the index, the various factors including acetylated reagent amounts, reaction temperature and reaction time was optimized to determine the best acetylation process parameters of LLPs by single factor experiment and Response Surface Method (RSM). Furthermore, the characterization of Ac-LLPs and LLPs was preliminarily performed by Fourier Transform Infrared spectroscopy (FT-IR). Finally, the antioxidant activity of Ac-LLPs and LLPs was also evaluated compared with LLPs in vitro. Results from this study will give scientific foundations for Ac-LLPs preparation, which will expand their potential applications in the pharmaceutical and functional food industries.

\section{Materials and Methods}

\section{Materials}

Loquat leaves was purchased from Bozhou, Anhui province, China. DEAE-Sepharose CL-6B and Sephadex G-100 were purchased from GE Healthcare Life Science (Piscataway, NJ, USA). 1,1-Diphenyl-2-Picrylhydrazyl (DPPH) was bought from Sigma Aldrich (Sigma, St Louis, MO, USA). 2-Amino-2-hydroxymethyl-1,3propanediol (Tris) was purchased from Solarbio (Beijing, China). Acetic anhydride, ferrous sulfate, hydrogen peroxide, salicylic acid and other chemicals used in this study were of analytical reagent grade.

\section{Preparation of LLPS}

Loquat leaves were washed and cut into square pieces, then dried in the oven at $45^{\circ} \mathrm{C}$. The dried loquat leaves were crushed into powder and passed through a 60-mesh sieve.

LLPs were prepared by the hot water extraction method (Fu et al., 2019). Briefly, the powder was mixed with hot water at a ratio of $1: 15(\mathrm{~g} / \mathrm{mL})$ and extracted at $70^{\circ} \mathrm{C}$ for $2 \mathrm{~h}$. The residue was removed by centrifugation (4500 rpm, $15 \mathrm{~min}$ ). The supernatant was condensed to $1 / 3$ of its original volume and deproteinated by the sevage method. Four volume of ethanol were added to precipitate crude polysaccharides at $4^{\circ} \mathrm{C}$ for $24 \mathrm{~h}$. After centrifugation (4500 rpm, $15 \mathrm{~min}$ ), the crude LLPs was obtained by freeze-drying.

The crude LLPs was dissolved in distilled water and filtered through a $0.45 \mu \mathrm{m}$ micro membrane filter. The filtrate was eluted with a linear gradient sodium chloride solution $(0-1.5 \mathrm{M})$ at a flow rate of $2.0 \mathrm{~mL} / \mathrm{min}$ on a DEAE-Sepharose CL-6B column $(2.6 \times 30 \mathrm{~cm})$ by a GE AKTA purifier system (UPC-100, USA). The major fraction was collected and further eluted with $0.05 \mathrm{~mol} / \mathrm{L}$ sodium chloride on a Sephadex G-100 gel chromatography column $(2.6 \times 160 \mathrm{~cm})$. The purified LLPs was obtained and freeze-dried for further study.

Determination of Degree of Substitution (DS) for Ac-LLPs

$0.1 \mathrm{~g}$ of Ac-LLPs was dissolved in $10 \mathrm{~mL} \mathrm{NaOH}$ solutions $(0.01 \mathrm{M})$. After adding two drops of phenolphthalein indicator, the mixtures were titrated with $\mathrm{HCl}$ solutions $(0.01 \mathrm{M})$. The volume of $\mathrm{HCl}$ solutions used for titration was recorded. DS for Ac-LLPs was measured based on the reported method previously (Das et al., 2010), which was calculated as follows:

$$
\begin{aligned}
& \text { Acetyl group content }(A c, \%) \\
& =\left[\left(V_{\mathrm{NaOH}} \times C_{\mathrm{NaOH}}-V_{\mathrm{HCl}} \times C_{\mathrm{HCl}}\right) \times 43\right] / m \times 100 \% \\
& D S=1.62 \times A c /(43-0.42 \times A c)
\end{aligned}
$$

\section{Single Factor Experiment of Ac-LLPs Preparation}

The acetylation of LLPs was prepared according to the reported method (Ma et al., 2012), which was displayed in Fig. 1.

\section{Effect of Acetylated Reagent Amounts on DS of Ac- LLPS}

LLPs $(5 \mathrm{~g})$ were dissolved in $500 \mathrm{~mL}$ distilled water and the $\mathrm{pH}$ was adjusted to 11.0 with $\mathrm{NaOH}$ solution (5 M). Then different amounts (1.0, 1.5, 2.0, 2.5, 3.0, 4.0, 5.0 $\mathrm{mL}$ ) of acetylated reagent (acetic anhydride) were added in the mixtures and reacted at $30^{\circ} \mathrm{C}$ for $2 \mathrm{~h}$, respectively. 


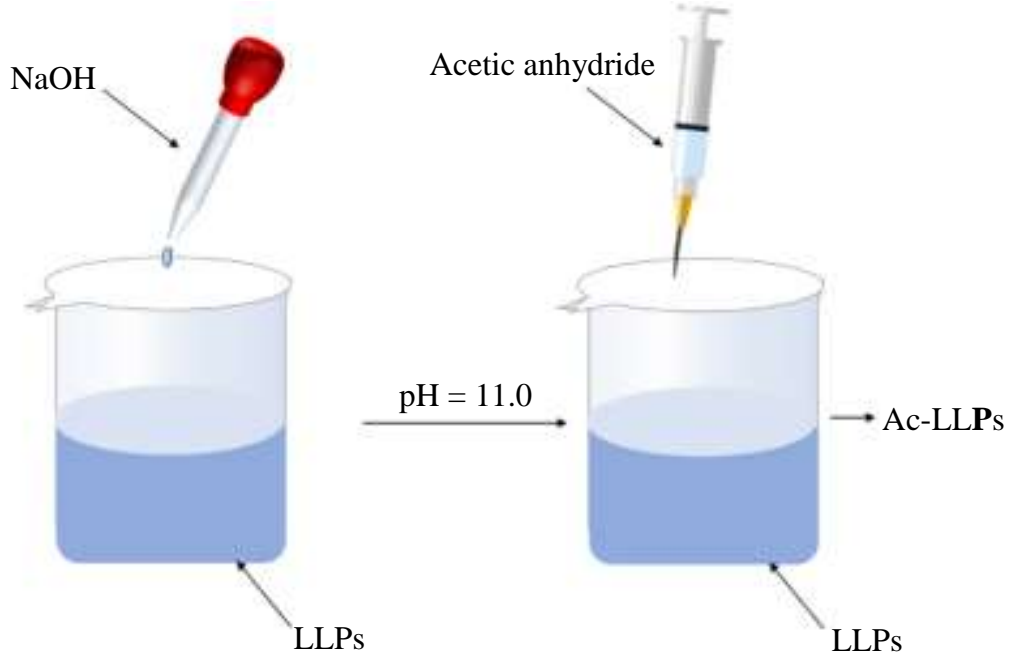

Fig. 1: Schematic illustration for acetylation process of LLPs

Table 1: Factors and levels designed for response surface analysis

\begin{tabular}{llrr} 
& & Levels & \\
& & -1 & 0 \\
\hline A & Factors & -1 & 2.5 \\
B & Acetylated reagent amounts $(\mathrm{mL})$ & 2.0 & 2.0 \\
$\mathrm{C}$ & Time $(\mathrm{h})$ & 1.5 & 3.0 \\
\hline
\end{tabular}

\section{Effect of Different Reaction Time on DS of Ac-LLPs}

LLPs $(5 \mathrm{~g})$ were dissolved in $500 \mathrm{~mL}$ distilled water and the $\mathrm{pH}$ was adjusted to 11.0 with $\mathrm{NaOH}$ solution $(5 \mathrm{M})$. $2.0 \mathrm{~mL}$ of acetic anhydride was added in the mixtures and reacted at $30^{\circ} \mathrm{C}$ for different time $(1.0,1.5,2.0,2.5$, 3.0, 4.0, 5.0 h), respectively.

\section{Effect of Different Reaction Temperature on DS of Ac-LLPs}

LLPs $(5 \mathrm{~g})$ was dissolved in $500 \mathrm{~mL}$ distilled water and the $\mathrm{pH}$ was adjusted to 11.0 with $\mathrm{NaOH}$ solution $(5 \mathrm{M})$. $2.0 \mathrm{~mL}$ of acetic anhydride was added in the mixtures and reacted at different temperature $(10,20,25,30,35$, $40,50,60,70^{\circ} \mathrm{C}$ ) for $2 \mathrm{~h}$, respectively.

After acetylated reaction, the solution was neutralized to 7.0 with $\mathrm{HCl}$ solution $(0.1 \mathrm{M})$ to terminate the reaction and dialyzed (molecular weight cut off: 3500 Da) against distilled water for $48 \mathrm{~h}$. The aqueous solution was precipitated with four volume of ethanol at $4^{\circ} \mathrm{C}$ for $24 \mathrm{~h}$. The resulting precipitate was freeze-dried to obtain Ac-LLPs.

\section{Experiment Design of Response Surface Method}

The ranges of the factors were determined according to the results of single factor experiment. DS was used as the response value, a Box-Behnken experimental design with three factors and three levels was chosen to optimize the acetylation modification of Ac-LLPs. Factors and levels designed for response surface method were shown in Table 1, including acetylated reagent amounts $(2.0,2.5,3.0 \mathrm{~mL})$, reaction time $(1.5,2.0,2.5 \mathrm{~h})$ and reaction temperature $\left(25,30,35^{\circ} \mathrm{C}\right)$.

\section{FT-IR Spectroscopy}

The powders of LLPs and Ac-LLPs were fully dried. Then the dried samples were mixed with potassium bromide (spectroscopic grade) and pressed into pellets for spectrometric measurement, respectively. The FT-IR spectra of LLPs and Ac-LLPs were recorded and analyzed on a Thermo Scientific Nicolet 6700 infrared spectrophotometer (USA) at the frequency range of $400-4000 \mathrm{~cm}^{-1}$ (Fu et al., 2019).

\section{DPPH Radical Scavenging Activity}

The ability of polysaccharides on scavenging DPPH radical was determined according to the previous method ( $\mathrm{Li}$ and Shah, 2014). $2.0 \mathrm{~mL}$ of aqueous polysaccharides at various concentrations $(0.2,0.4,0.6,0.8,1.0 \mathrm{~g} / \mathrm{L})$ were mixed with $2.0 \mathrm{~mL}$ of DPPH radical $(0.1 \mathrm{~mol} / \mathrm{L})$ or ethanol and incubated for $30 \mathrm{~min}$ at $30^{\circ} \mathrm{C}$ in dark. The absorbance of the mixture was measured at $517 \mathrm{~nm}$. Distilled water was used as the blank control instead of polysaccharide solution. The scavenging ability on DPPH radical was calculated by the following equation: 
The DPPH radical scavenging activity $(\%)$

$$
=\left[1-\left(A_{1}-A_{2}\right) / A_{0}\right] \times 100 \%
$$

where, $A_{1}$ was the absorbance values of aqueous polysaccharides at various concentrations. $A_{2}$ was the absorbance values of the mixture without DPPH. $A_{0}$ was the absorbance values of the mixture without aqueous polysaccharides.

\section{Hydroxyl Radical Scavenging Activity}

The hydroxyl radical scavenging activities of polysaccharides were carried out according to the previous method ( $\mathrm{Li}$ and Shah, 2014). Briefly, the reaction mixture contained $1.0 \mathrm{~mL}$ salicylic acid ethanol solution $(9.0 \mathrm{mM})$ and $1.0 \mathrm{~mL} \mathrm{FeSO}_{4}$ solution $(9.0 \mathrm{mM})$ and $1.0 \mathrm{~mL} \mathrm{H}_{2} \mathrm{O}_{2}$ solution $(8.8 \mathrm{mM})$. After adding $1.0 \mathrm{~mL}$ aqueous polysaccharides at various concentrations $(0.2,0.4,0.6,0.8,1.0 \mathrm{~g} / \mathrm{L})$, the mixture was incubated for $30 \mathrm{~min}$ at $37^{\circ} \mathrm{C}$. The absorbance was measured at $510 \mathrm{~nm}$. The hydroxyl radical scavenging activity was calculated by the formula:

$$
\begin{aligned}
& \text { The hydroxyl radical scavenging activity }(\%) \\
& =\left[1-\left(A_{1}-A_{2}\right) / A_{0}\right] \times 100 \%
\end{aligned}
$$

where, $A_{1}$ was the absorbance values of aqueous polysaccharides at various concentrations. $A_{2}$ was the absorbance values of the mixture without $\mathrm{H}_{2} \mathrm{O}_{2} . A_{0}$ was the absorbance values of distilled water instead of aqueous polysaccharides.

\section{Superoxide Anion Radical Scavenging Activity}

The superoxide anion radical scavenging activities of polysaccharides were carried out according to the reported method (Li and Shah, 2014). Briefly, $1.0 \mathrm{~mL}$ of aqueous polysaccharides at various concentrations $(0.2$, $0.4,0.6,0.8,1.0 \mathrm{~g} / \mathrm{L}), 4.5 \mathrm{~mL}$ of tris- $\mathrm{HCl}$ buffer solution (50 mM, pH 8.2) and $3.2 \mathrm{~mL}$ distilled water were mixed and stood for $20 \mathrm{~min}$ at $25^{\circ} \mathrm{C}$. Then $0.3 \mathrm{~mL}$ of pyrogallol ( $7 \mathrm{mM}$ ) was added to incubate for $3 \mathrm{~min}$ at $25^{\circ} \mathrm{C}$. The absorbance value was measured at $325 \mathrm{~nm}$. Distilled water was used as the blank control instead of polysaccharide solutions. The superoxide anion radical scavenging activity was calculated by the formula:

$$
\begin{aligned}
& \text { The superoxide anion radical scavenging activity }(\%) \\
& =\left(1-A / A_{0}\right) \times 100 \%
\end{aligned}
$$

where, $A$ was the absorbance values of aqueous polysaccharides at various concentrations. $A_{0}$ was the absorbance values of distilled water instead of aqueous polysaccharides.

\section{Statistical Analysis}

The experimental data and analysis were evaluated with a Design Expert ${ }^{\circledR}$ software and Analysis Of Variance (ANOVA). Tukey's test was used to evaluate the significant differences, with $p<0.05$ defined significant.

\section{Results}

\section{Effect of Process Parameters}

Acetylation can improve the physicochemical properties and biological activities of polysaccharides, in which DS is an important factor (Xie et al., 2015; Song et al., 2013). To confirm the optimal acetylation process of LLPs, the DS was used as the index to explore from three aspects of acetylated reagent amounts, reaction time and temperature. As displayed in Fig. 2A, with the increase of acetic anhydride amount in the acetylated reaction of LLPs, there was a trend of first increasing and then decreasing for DS. When the amount of acetic anhydride was $2.5 \mathrm{~mL}$, DS reached the maximum value of 2.654. Therefore, $2.5 \mathrm{~mL}$ was selected as the suitable acetylated reagent amounts.

The effect of time on the DS was shown in Fig. 2B. When the reaction time was $2 \mathrm{~h}$, the DS increased to the highest value and then decreased slowly. Thus, the results indicated that the suitable reaction time was $2 \mathrm{~h}$.

As another key factor, the reaction temperature was also tested shown in Fig. 2C, the DS increased up to a peak at $30^{\circ} \mathrm{C}$ and then declined. When the temperature exceeded $30^{\circ} \mathrm{C}$, excessive temperature would easily accelerate the hydrolysis rate of acetic anhydride in the reaction system. So, $30^{\circ} \mathrm{C}$ was selected as the suitable reaction temperature.

\section{Box-Behnken Design and Analysis}

\section{Statistical Analysis and Model Fitting}

Based on single factor experimental results, the acetylation process of LLPs was further optimized by Box-Behnken design software. As shown in Table 2. The whole design consisted of 17 experimental points with 5 center points carried out in random order. The experimental data was analyzed by multiple regression, the DS was predicted by the following second order polynomial equation:

$$
\begin{aligned}
& \mathrm{Y}=-2.43649+0.43162 A+0.16349 B-0.18308 C \\
& +0.019309 A B+0.31910 A C+0.017540 B C \\
& -0.43798 A^{2}-3.97280 \times 10^{-3} B^{2}-0.20308 C^{2}
\end{aligned}
$$

where, $Y$ was DS; $A, B$ and $C$ represented acetylated reagent amounts $(\mathrm{mL})$, time $(\mathrm{h})$, temperature $\left({ }^{\circ} \mathrm{C}\right)$, respectively. 

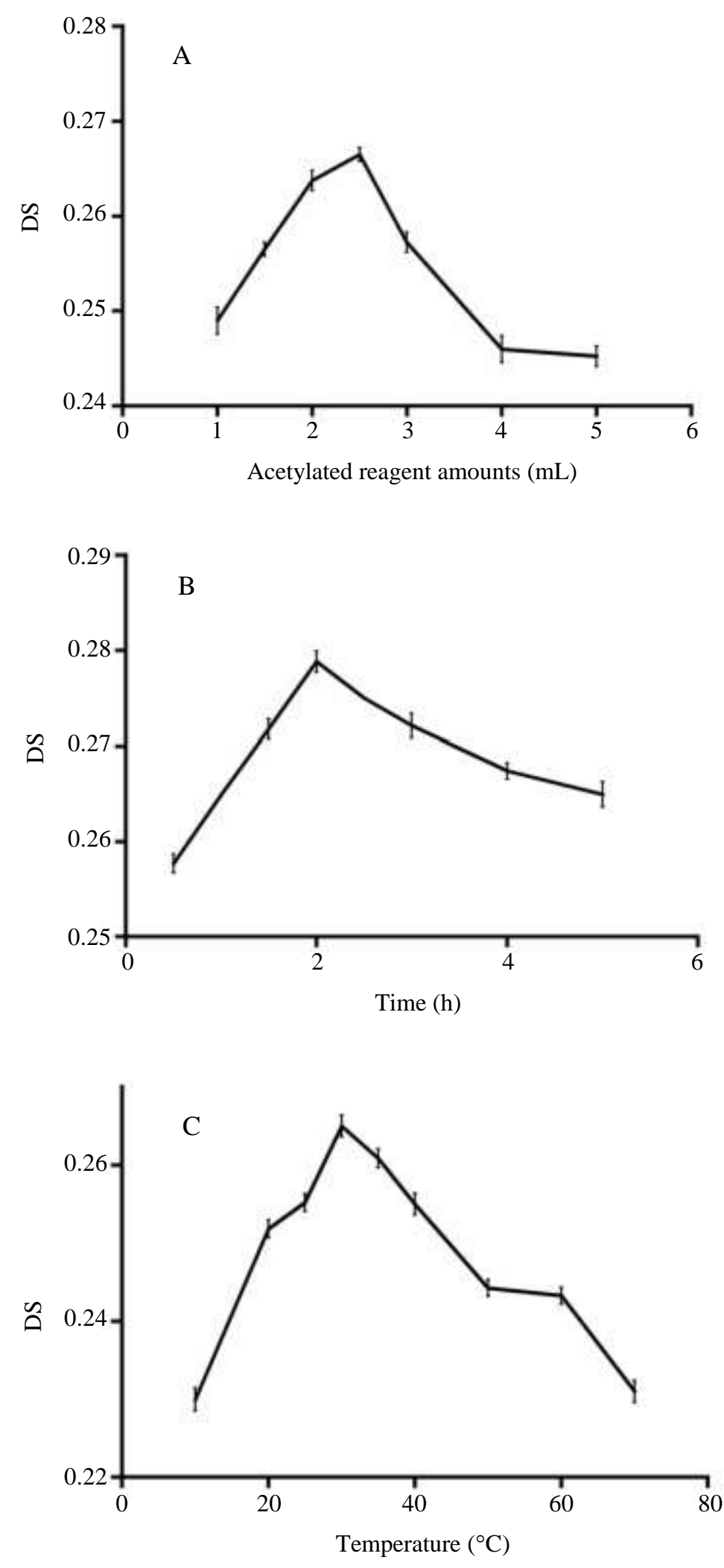

Fig. 2: Effect of acetylated reagent amounts (A), time (B) and temperature (C) on the DS of Ac-LLPs. Values were presented as means $\pm \mathrm{SD}(n=3)$ 
Table 2: The Box-Behnken design matrix with response values for DS of Ac-LLPs

\begin{tabular}{|c|c|c|c|c|}
\hline \multirow[b]{2}{*}{ Runs } & \multicolumn{3}{|c|}{ Factors } & \multirow[b]{2}{*}{ DS } \\
\hline & $A$ & $B$ & $C$ & \\
\hline 1 & 3.00 & 2.00 & 35.00 & 0.2803 \\
\hline 2 & 2.50 & 2.00 & 30.00 & 0.2956 \\
\hline 3 & 2.00 & 2.00 & 35.00 & 0.2074 \\
\hline 4 & 2.50 & 2.50 & 25.00 & 0.0905 \\
\hline 5 & 2.50 & 1.50 & 35.00 & 0.0958 \\
\hline 6 & 3.00 & 2.00 & 25.00 & 0.1056 \\
\hline 7 & 2.50 & 2.50 & 35.00 & 0.2548 \\
\hline 8 & 2.50 & 2.00 & 30.00 & 0.3512 \\
\hline 9 & 2.50 & 2.00 & 30.00 & 0.3275 \\
\hline 10 & 2.00 & 1.50 & 30.00 & 0.2685 \\
\hline 11 & 2.50 & 2.00 & 30.00 & 0.3627 \\
\hline 12 & 2.50 & 2.00 & 30.00 & 0.4152 \\
\hline 13 & 2.00 & 2.50 & 30.00 & 0.1652 \\
\hline 14 & 3.00 & 2.50 & 30.00 & 0.2714 \\
\hline 15 & 2.00 & 2.00 & 25.00 & 0.2018 \\
\hline 16 & 2.50 & 1.50 & 25.00 & 0.1254 \\
\hline 17 & 3.00 & 1.50 & 30.00 & 0.0556 \\
\hline
\end{tabular}

Table 3: ANOVA for response surface quadratic model

\begin{tabular}{llllcl}
\hline Source & Sum of squares & df & Mean squares & F-value & P-value \\
\hline Model & 0.18 & 9 & 0.020 & 15.29 & $0.0008^{* *}$ \\
$A$ & $2.113 \times 10^{-3}$ & 1 & $2.113 \times 10^{-3}$ & 1.64 & 0.2413 \\
$B$ & $6.997 \times 10^{-3}$ & 1 & $6.997 \times 10^{-3}$ & 5.43 & 0.02 \\
$C$ & 0.012 & 1 & 0.012 & 19.75 & $0.0173^{*}$ \\
$A B$ & 0.025 & 1 & 0.025 & 5.54 & $0.0030^{* *}$ \\
$A C$ & $7.149 \times 10^{-3}$ & 1 & $7.149 \times 10^{-3}$ & 7.29 & 0.0507 \\
$B C$ & $9.399 \times 10^{-3}$ & 1 & $9.399 \times 10^{-3}$ & 38.59 & $0.0306^{*}$ \\
$A^{2}$ & 0.011 & 1 & 0.011 & 32.73 & $0.0215^{*}$ \\
$B^{2}$ & 0.050 & 1 & 0.050 & $0.0004 * *$ \\
$C^{2}$ & 0.042 & 1 & 0.042 & 0.19 & $0.0007 * *$ \\
Residual & $9.025 \times 10^{-3}$ & 7 & $1.289 \times 10^{-3}$ & & 0.8955 \\
Lake of fit & $1.146 \times 10^{-3}$ & 3 & $3.821 \times 10^{-4}$ & & \\
Pure error & $7.878 \times 10^{-3}$ & 4 & $1.970 \times 10^{-4}$ & & \\
Cor total & 0.19 & 16 & & & \\
$\mathrm{R}^{2}$ & 0.9516 & & & & \\
$\mathrm{R}^{2}$ adj & 0.8894 & & & &
\end{tabular}

Notes: * indicated significant difference $(p<0.05)$; ** indicated extremely significant difference $(p<0.01)$

The statistical significance of the regression model was tested using F-test and P-value. The ANOVA for the response surface quadratic model was summarized in Table 3. The ideal regression equation and the high model significance was confirmed by its high $\mathrm{F}$-value (15.29) and low P-value $(p<0.01)$. The goodness-of-fit and reliability could be testified by the determination coefficient $\left(\mathrm{R}^{2}=0.9516\right)$, adjusted determination coefficient $\left(\mathrm{R}^{2}\right.$ adj $\left.=0.8894\right)$ and the coefficient of the variation $($ C.V. $=9.28 \%)$, respectively. Lake of fit was not significant $(p=0.9171)$, which indicated that other factors have little interference to the DS of Ac-LLPs. These results clearly showed that the model was credible for the experimental data, which provided the theoretical basis for the acetylation of LLPs.

\section{Optimization of Acetylation Process of LLPS}

The 3D response surfaces of factor interactions were shown in Fig. 3, which reflected the relationship between two independent variables. According to the tightness of the contour line and the smoothness of the curve, the influence order of single factor on the DS of Ac-LLPs was $C>B>A$. Figure 3 a represented the effects of acetylated reagent amounts $(A)$, time $(B)$ and their interactions on the DS of Ac-LLPs. Along with acetylated reagent amounts $(A)$ from 2.58 to 3.0 $\mathrm{mL}$, a linearly decrease of DS was produced with the increasing of time $(B)$. The DS of Ac-LLPs reached the maximum value of 0.3588 . Their interaction between acetylated reagent amounts $(A)$ and time $(B)$ 
was extremely significant $(p<0.01)$. In Fig. $3 b$, the DS of Ac-LLPs increased when acetylated reagent amounts $(A)$ and temperature $(C)$ increased in the ranges of 2 to $2.58 \mathrm{~mL}$ and 25 to $31.48^{\circ} \mathrm{C}$, respectively. Figure $3 \mathrm{c}$ showed the peak DS was obtained when time $(B)$ and temperature $(C)$ were 2.13 $\mathrm{h}$ and $31.48^{\circ} \mathrm{C}$, respectively. The interactions between $A B, B C$ were significant.

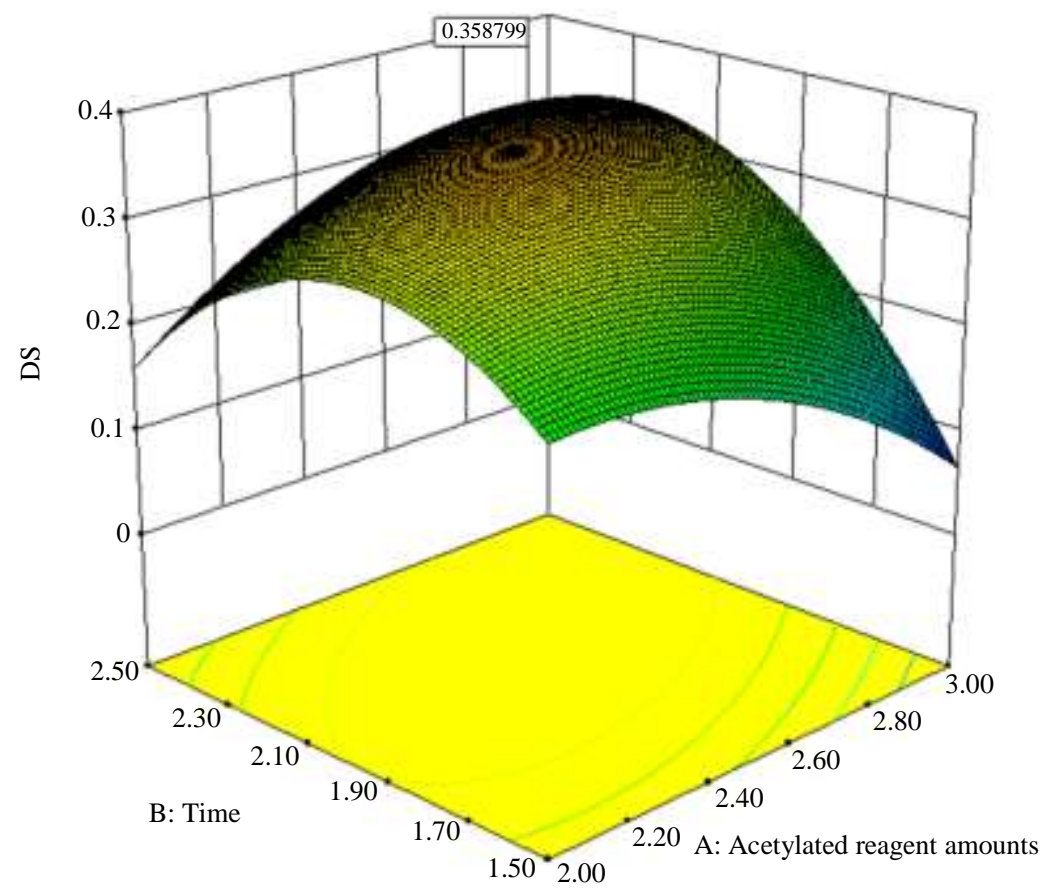

(a)

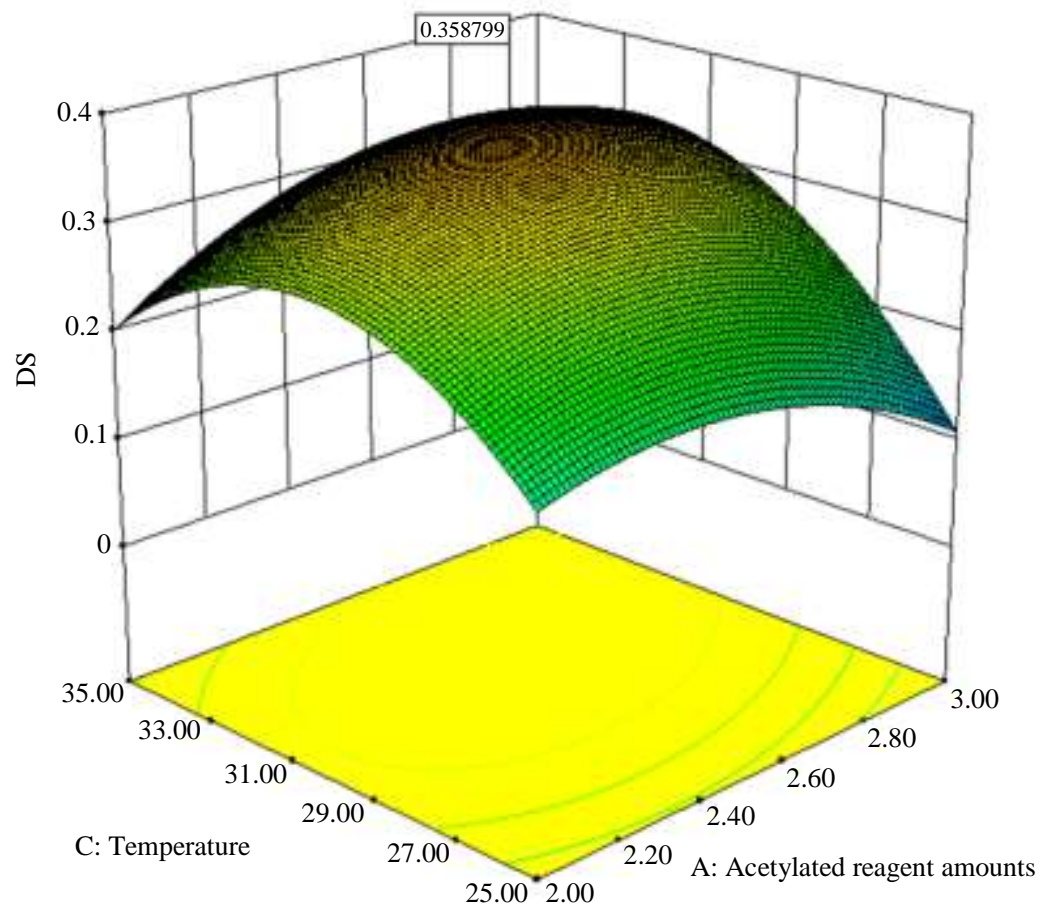

(b) 


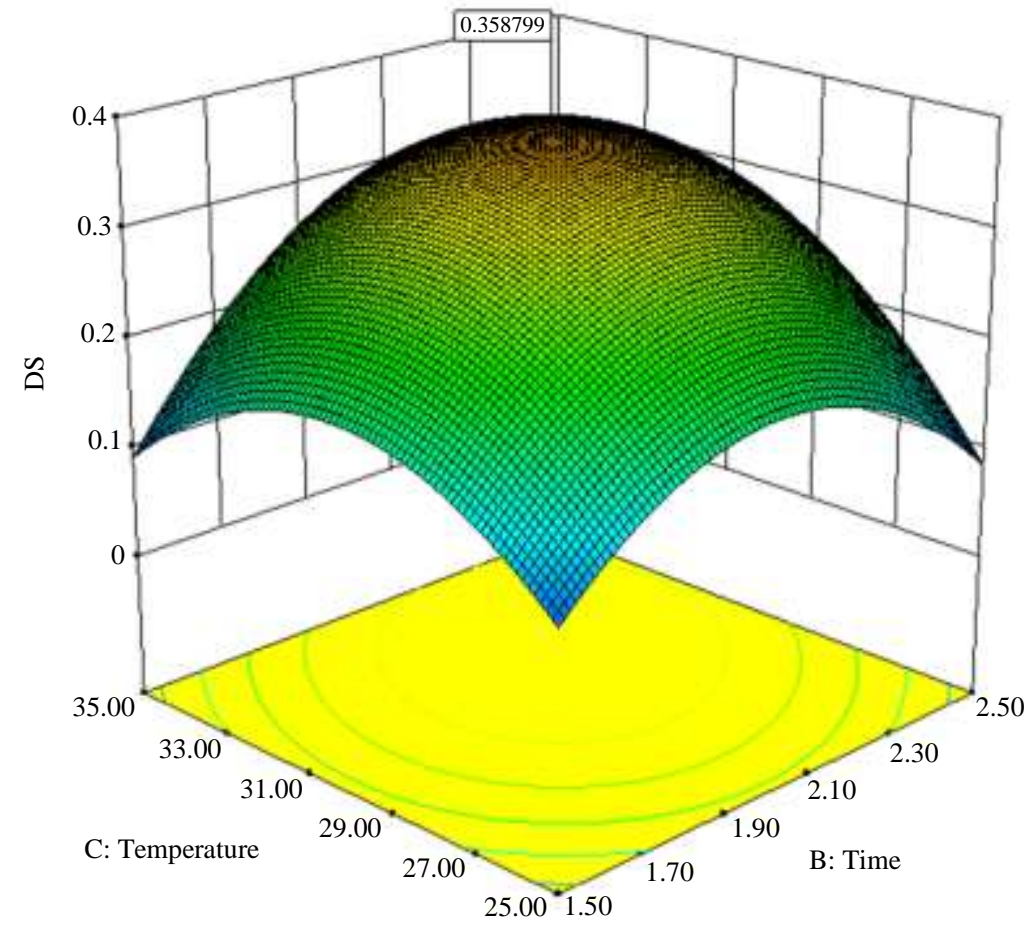

(c)

Fig. 3: Response surface plots showing the effects of acetylated reagent amounts $(A)$, time $(B)$ and temperature $(C)$ on the DS of AcLLPs

\section{Confirmative Tests}

Through the Box-Behnken design software, the optimum process of LLPs acetylation was obtained as following: Acetylated reagent amounts (acetic anhydride) were $2.58 \mathrm{~mL}$, reaction time was $2.13 \mathrm{~h}$, reaction temperature was $31.48^{\circ} \mathrm{C}$, respectively. Under this acetylated condition, the predicted value of DS for Ac-LLPs was 0.3588. In view of the practical operation, the parameters were modified as follows: Acetylated reagent amounts (acetic anhydride) of $2.60 \mathrm{~mL}$, reaction time of $2.10 \mathrm{~h}$, reaction temperature of $31^{\circ} \mathrm{C}$ in validation experiments. The actual experimental DS of Ac-LLPs had reached $0.3570 \pm 0.005(n=3)$, which suggested this model could be used as the prediction of DS in LLPs acetylation totally.

\section{Characterization of LLPs and Ac-LLPS}

FT-IR spectra is a supplementary technique for the identification of characteristic functional groups of polysaccharides in the range from 4000 to $500 \mathrm{~cm}^{-1}$. At around $3450 \mathrm{~cm}^{-1}$, the strong absorption peaks could be attributed to the stretching vibration of $\mathrm{O}-\mathrm{H}$ from hydroxyl and carboxyl groups (Qiu et al., 2016). At around 1600 and $1400 \mathrm{~cm}^{-1}$, the band corresponded to the $v_{\mathrm{COO}}$ (asymmetric) and $v_{\mathrm{COO}^{-}}$(symmetric) of carboxyl group in uronic acid structure, respectively (Bukzem et al., 2016). At around
$1000 \mathrm{~cm}^{-1}$, the absorption bands were contributed by the vibrations of pyranoid rings (Cheng et al., 2016).

The FT-IR spectra of LLPs and Ac-LLPs was shown in Fig. 4. The general profile of the spectra for LLPs and AcLLPs was similar. However, there were still some differences. One increased absorption intensity exhibited at $1725 \mathrm{~cm}^{-1}$, which was mainly caused by the stretching vibration of $\mathrm{C}=\mathrm{O}$ from ester group. At $1250 \mathrm{~cm}^{-1}$, another increased absorption intensity was attributed to the stretching vibration of $\mathrm{C}-\mathrm{O}$ from ester group. The vibration peak is related to the acetyl group, which suggested the existence of acetyl group (Zhang et al., 2010). Hence, the acetylation of LLPs has been successfully modified.

\section{Antioxidant Activities of LLPs and Ac-LLPS}

Generally, the DPPH free radical scavenging capacity is chosen as the evaluation index of antioxidant activity of natural active ingredients. In Fig. 5A, LLPs and Ac-LLPs had the reducing power on DPPH free radical in a concentration dependent manner. After acetylated modification, the scavenging capacity of Ac-LLPs on DPPH free radical enhanced greatly compared with LLPs. However, the reducing power of both samples were lower than positive control $(\mathrm{Vc})$. At a concentration of 1.0 $\mathrm{mg} / \mathrm{mL}$, the scavenging rates of LLPs, Ac-LLPs and $\mathrm{Vc}$ were $38.72,63.65$ and $92.05 \%$, respectively. Moreover, the addition of acetyl group had a significantly lifting effect on the DPPH free radical scavenging activity. 


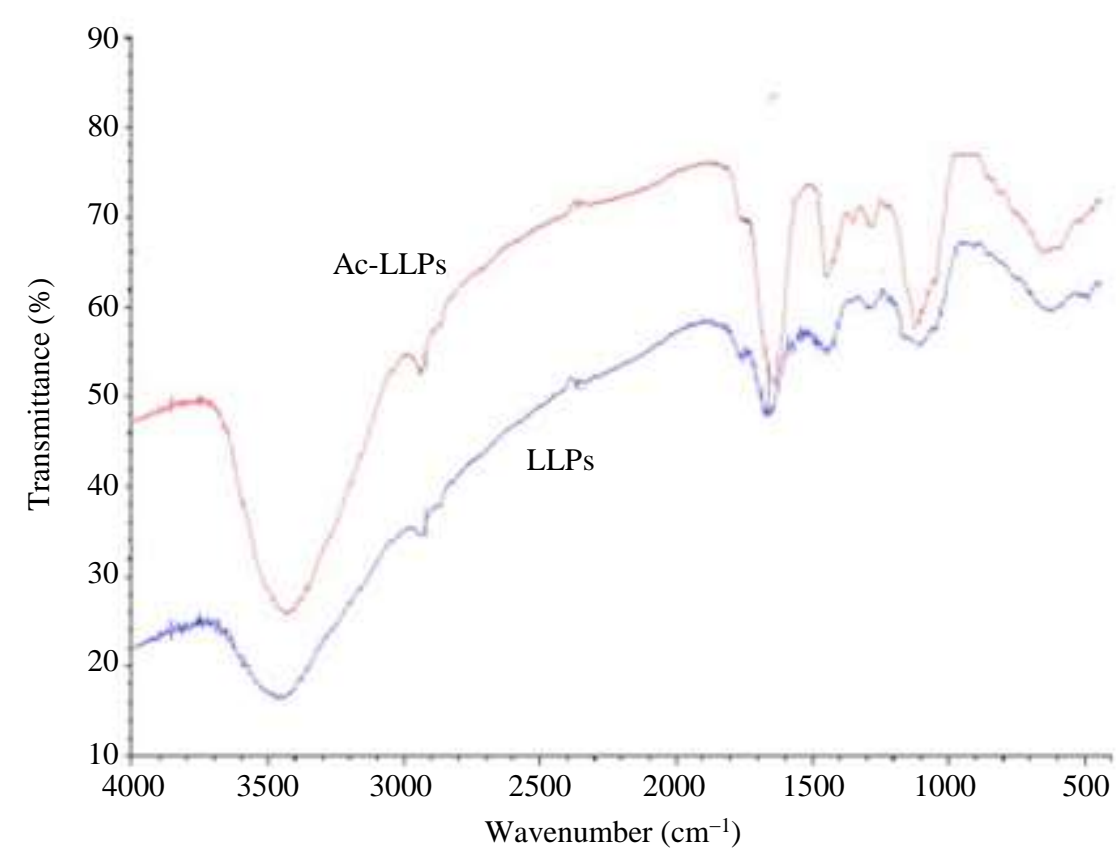

Fig. 4: FT-IR spectrograms of LLPs and Ac-LLPs
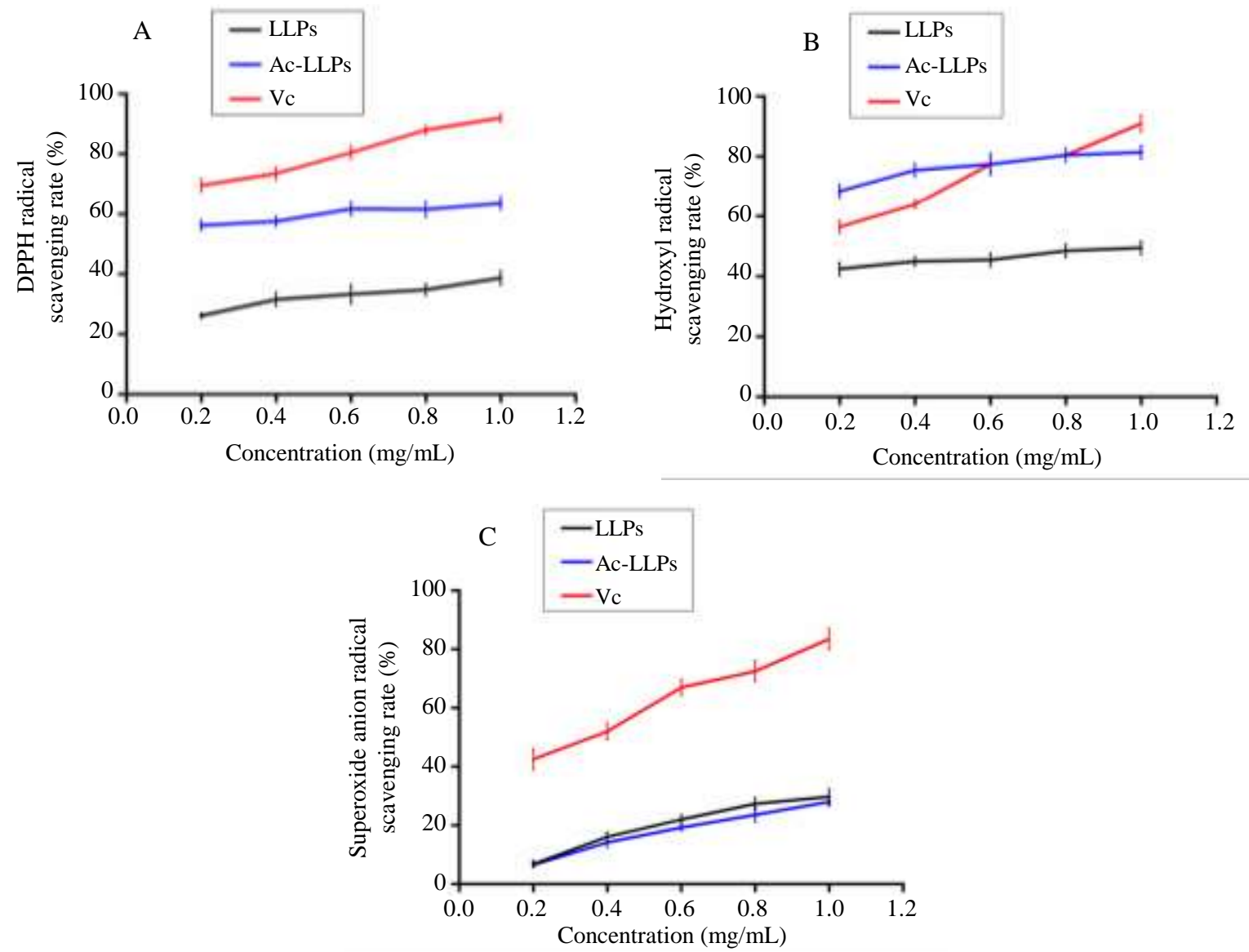

Fig. 5: The scavenging effect of LLPs and Ac-LLPs on DPPH radical (A), hydroxyl radical (B) and superoxide anion radical (C). Vitamin $\mathrm{C}(\mathrm{Vc})$ was chosen as a positive drug and values were presented as means $\pm \operatorname{SD}(n=3)$ 
In addition, the body produces ROS during its metabolism, including superoxide anion radical, hydroxyl radical (Lushchak, 2014). When these free radicals accumulate to a certain concentration, they will cause damage to normal life activities of cells in the body (Forman and Torres, 2002). As displayed in Fig. 5B, with the increase of concentration from 0.2 to $1 \mathrm{mg} / \mathrm{mL}$, LLPs and Ac-LLPs showed dose-dependent effects on the reducing power of hydroxyl radical. Consistent with the results of scavenging DPPH free radical, the scavenging capacity of Ac-LLPs was stronger than LLPs. At a concentration of $1.0 \mathrm{mg} / \mathrm{mL}$, the scavenging rate of LLPs, Ac-LLPs and Vc reached the maximum of $49.52,81.57$ and $91.03 \%$, respectively.

In Fig. 5C, LLPs and Ac-LLPs had dose-dependent effects on scavenging superoxide anion radical. However, both samples gave a completely different results on the reducing power of superoxide anion radical. In the concentration from 0.2 to $1.0 \mathrm{mg} / \mathrm{mL}$, the scavenging capacity of LLPs was slightly higher than AcLLPs. When the concentration was $1.0 \mathrm{mg} / \mathrm{mL}$, the maximum of LLPs, Ac-LLPs and Vc scavenging rate achieved $29.83,28.11$ and $83.46 \%$, respectively.

\section{Discussion}

Studies on the structural-activity relationship of polysaccharides showed that the antioxidant activity of polysaccharides may come from the common hydroxyl groups, phenolic substances and cross-linked proteins in their sugar rings (Siu et al., 2014). The antioxidant mechanism of polysaccharides is relatively complex, which may be related to the type and higher structure of its binding components. After acetylated modification of polysaccharides, its spatial conformation changes and provides hydroxyl easily. Thus, acetylated polysaccharides are beneficial to capture free radicals and improve antioxidant activity.

In this study, LLPs was proved to possess strong antioxidant activities. Moreover, the introduction of acetyl group can indeed improve the antioxidant activity of Ac-LLPs. But the insertion of acetyl group had no obvious influences on scavenging superoxide anion radical ability for LLPs. Similar with this study, (Song et al., 2013) found that the scavenging ability of the acetylated pumpkin polysaccharide was higher than that of itself on the DPPH radical and hydroxyl radical. In addition, (Zhang et al., 2014) confirmed that the scavenging activity of acetylated green seaweed polysaccharide on the hydroxyl radical was positively correlated with the DS. This may be related to the reduction of hydrogen bonds and the activation of hydrogen atoms on heterocarbons in polysaccharides, which enhances the ability of polysaccharides to provide hydrogen atoms and thus terminates the chain reaction of free radicals after the acetylation of polysaccharides (Li et al., 2016; Song et al., 2013).

\section{Conclusion}

Oxidative stress is closely related with chronic diseases (arteriosclerosis, cancer and diabetes mellitus). Chronic diseases are usually caused by excessive production of ROS. Acetylated polysaccharides have been proved to have the excellent capability to capture free radicals and improve antioxidant activity, which are expected to become a new food additive or antioxidant supplement. In this study, LLPs was successfully isolated and purified from loquat leaves by DEAE-Sepharose CL6B and Sephadex G-200 column chromatography. Then Ac-LLPs was synthesized with LLPs as raw materials by the acetic anhydride method. Through RSM and single factor experiments, the various factors including acetylated reagent amounts, reaction temperature and reaction time were optimized with DS as the index. The acetylation process parameters of LLPs were obtained as following: Acetylated reagent amounts was $2.60 \mathrm{~mL}$, reaction time was $2.10 \mathrm{~h}$, reaction temperature was $31^{\circ} \mathrm{C}$. The highest value of DS was $0.3570(n=3)$. In addition, FT-IR spectra proved acetyl group in Ac-LLPs was successfully added. Moreover, compared with LLPs, AcLLPs had strong scavenging effect on DPPH free radicals and hydroxyl free radicals and weak scavenging effect on superoxide anion, which may be caused by hydrogen bond reduction and hydrogen atom activation on heterocarbons in Ac-LLPs. The results suggested that acetylated modification could indeed significantly enhance the antioxidant activity of LLPs, which was beneficial to broaden its potential applications in the pharmaceutical and functional food industries. Furthermore, the present study will give scientific foundations for Ac-LLPs preparation, which can provide reference for acetylation preparation of other polysaccharides.

\section{Acknowledgement}

This research was supported in part by programs of Research Startup Fund Project of Changshu Institute of Technology (KYZ2016092Z) and college student innovation and entrepreneurship training program project (XJXZ2020394).

\section{Author's Contributions}

Yiting Xue and Ze Zhang: Participated in all experiments and article writing.

Xiao Ding and Xinyi Wu: Participated in acetylation process of LLPs and data analysis.

Xiaolong Cao, Weizhi Zhang and Jiehan Zhang: Participated in characterization, antioxidant activities of Ac-LLPs and data processing. 
Yiyong Chen: Participated in project design and experimental guidance of this manuscript.

\section{Ethics}

This article is original and contains unpublished material. The corresponding author confirms that all of the other authors have read and approved the manuscript and no ethical issues involved.

\section{Reference}

Agsalda-Garcia, M., Shieh, T., Souza, R., Kamada, N., Loi, N., Oda, R., \& Shiramizu, B. (2020). RamanEnhanced Spectroscopy (RESpect) Probe for Childhood Non-Hodgkin Lymphoma. SciMedicine Journal, 2(1), 1-7.

Ananthi, S., Raghavendran, H. R. B., Sunil, A. G., Gayathri, V., Ramakrishnan, G., \& Vasanthi, H. R. (2010). In vitro antioxidant and in vivo antiinflammatory potential of crude polysaccharide from Turbinaria ornata (Marine Brown Alga). Food and chemical toxicology, 48(1), 187-192.

Beridze, M., Kalandia, A., Japaridze, I., Vanidze, M., Varshanidze, N., Turmanidze, N., \& Jakeli, E. (2020). Phytochemical Study of Endemic Species Helleborus Caucasicus and Helleborus Abchasicus. HighTech and Innovation Journal, 1(1), 28-32.

Bukzem, A. L., Signini, R., dos Santos, D. M., Lião, L. M., \& Ascheri, D. P. R. (2016). Optimization of carboxymethyl chitosan synthesis using response surface methodology and desirability function. International journal of biological macromolecules, 85, 615-624.

Cha, D. S., Eun, J. S., \& Jeon, H. (2011). Antiinflammatory and antinociceptive properties of the leaves of Eriobotrya japonica. Journal of Ethnopharmacology, 134(2), 305-312.

Chen, B., Long, P., Sun, Y., Meng, Q., Liu, X., Cui, H., \& Zhang, L. (2017). The chemical profiling of loquat leaf extract by HPLC-DAD-ESI-MS and its effects on hyperlipidemia and hyperglycemia in rats induced by a high-fat and fructose diet. Food \& function, 8(2), 687-694.

Chen, Y., Zhang, H., Wang, Y., Nie, S., Li, C., \& Xie, M. (2014). Acetylation and carboxymethylation of the polysaccharide from Ganoderma atrum and their antioxidant and immunomodulating activities. Food chemistry, 156, 279-288.

Cheng, Z., Zhang, Y., Song, H., Zhou, H., Zhong, F., Hu, H., \& Feng, Y. (2016). Extraction optimization, characterization and antioxidant activity of polysaccharide from Gentiana scabra bge. International journal of biological macromolecules, 93, 369-380.
Das, A. B., Singh, G., Singh, S., \& Riar, C. S. (2010). Effect of acetylation and dual modification on physico-chemical, rheological and morphological characteristics of sweet potato (Ipomoea batatas) starch. Carbohydrate Polymers, 80(3), 725-732.

Du, X., Zhang, J., Lv, Z., Ye, L., Yang, Y., \& Tang, Q. (2014). Chemical modification of an acidic polysaccharide (TAPA1) from Tremella aurantialba and potential biological activities. Food chemistry, 143, 336-340.

Forman, H. J., \& Torres, M. (2002). Reactive oxygen species and cell signaling: respiratory burst in macrophage signaling. American journal of respiratory and critical care medicine, 166, S4-S8.

Fu, Y., Yuan, Q., Lin, S., Liu, W., Du, G., Zhao, L., \& Li, D. Q. (2019). Physicochemical characteristics and biological activities of polysaccharides from the leaves of different loquat (Eriobotrya japonica) cultivars. International journal of biological macromolecules, 135, 274-281.

Lae, K. Z. W., Su, S. S., Win, N. N., Than, N. N., \& Ngwe, H. (2019). Isolation of Lasiodiplodin and Evaluation of some Biological Activities of the Stem Barks of Phyllanthus Albizzioides (Kurz) Hook. f. SciMedicine Journal, 1(4), 199-216.

Li, S., \& Shah, N. P. (2014). Antioxidant and antibacterial activities of sulphated polysaccharides from Pleurotus eryngii and Streptococcus thermophilus ASCC 1275. Food chemistry, 165, 262-270.

Li, S., Xiong, Q., Lai, X., Li, X., Wan, M., Zhang, J., \& Zhang, D. (2016). Molecular modification of polysaccharides and resulting bioactivities. Comprehensive Reviews in Food Science and Food Safety, 15(2), 237-250.

Liu, J., Luo, J., Ye, H., \& Zeng, X. (2012). Preparation, antioxidant and antitumor activities in vitro of different derivatives of levan from endophytic bacterium Paenibacillus polymyxa EJS-3. Food and Chemical Toxicology, 50(3-4), 767-772.

Liu, Y., Zhang, W., Xu, C., \& Li, X. (2016). Biological activities of extracts from loquat (Eriobotrya japonica Lindl.): a review. International journal of molecular sciences, 17(12), 1983.

Lu, X., Mo, X., Guo, H., \& Zhang, Y. (2012). Sulfation modification and anticoagulant activity of the polysaccharides obtained from persimmon (Diospyros kaki L.) fruits. International journal of biological macromolecules, 51(5), 1189-1195.

Lushchak, V. I. (2014). Free radicals, reactive oxygen species, oxidative stress and its classification. Chemico-biological interactions, 224, 164-175. 
Ma, L., Chen, H., Zhang, Y., Zhang, N., \& Fu, L. (2012). Chemical modification and antioxidant activities of polysaccharide from mushroom Inonotus obliquus. Carbohydrate polymers, 89(2), 371-378.

Papas, A. M. (1999). Antioxidant status, diet, nutrition and health. United Kingdom: CRC Press.

Qiu, J., Zhang, H., Wang, Z., Liu, S., \& Regenstein, J. M. (2016). Response surface methodology for the synthesis of an Auricularia auriculajudae polysaccharides-CDDP complex. International journal of biological macromolecules, 93, 333-343.

Shih, C. C., Lin, C. H., \& Wu, J. B. (2010). Eriobotrya japonica improves hyperlipidemia and reverses insulin resistance in high-fat-fed mice. Phytotherapy research, 24(12), 1769-1780.

Siu, K. C., Chen, X., \& Wu, J. Y. (2014). Constituents actually responsible for the antioxidant activities of crude polysaccharides isolated from mushrooms. Journal of Functional Foods, 11, 548-556.

Song, Y., Yang, Y., Zhang, Y., Duan, L., Zhou, C., Ni, Y., ... \& Hu, X. (2013). Effect of acetylation on antioxidant and cytoprotective activity of polysaccharides isolated from pumpkin (Cucurbita pepo, lady godiva). Carbohydrate polymers, 98(1), 686-691.

Trang, G. T. T., Linh, N. H., Linh, N. T. T., \& Kien, P. H. (2020). The Study of Dynamics Heterogeneity in $\mathrm{SiO} 2$ Liquid. HighTech and Innovation Journal, 1(1), 1-7.

Valko, M., Leibfritz, D., Moncol, J., Cronin, M. T., Mazur, M., \& Telser, J. (2007). Free radicals and antioxidants in normal physiological functions and human disease. The international journal of biochemistry \& cell biology, 39(1), 44-84.

Wang, J., Liu, L., Zhang, Q., Zhang, Z., Qi, H., \& Li, P. (2009). Synthesized oversulphated, acetylated and benzoylated derivatives of fucoidan extracted from Laminaria japonica and their potential antioxidant activity in vitro. Food Chemistry, 114(4), 1285-1290.

Wu, Y. X., Jian, T. Y., Lv, H., Ding, X. Q., Zuo, Y. Y., Ren, B. R., ... \& Li, W. L. (2018). Antitussive and expectorant properties of growing and fallen leaves of loquat (Eriobotrya japonica). Revista Brasileira de Farmacognosia, 28(2), 239-242.
Xie, J. H., Zhang, F., Wang, Z. J., Shen, M. Y., Nie, S. P., \& Xie, M. Y. (2015). Preparation, characterization and antioxidant activities of acetylated polysaccharides from Cyclocarya paliurus leaves. Carbohydrate Polymers, 133, 596-604.

Yang, X., Yang, S., Guo, Y., Jiao, Y., \& Zhao, Y. (2013). Compositional characterisation of soluble apple polysaccharides and their antioxidant and hepatoprotective effects on acute CCl4-caused liver damage in mice. Food Chemistry, 138(2-3), 1256-1264.

Zar, P. P. K., Yano, S., Sakao, K., Hashimoto, F., Nakano, T., Fujii, M., \& Hou, D. X. (2014). In vitro anticancer activity of loquat tea by inducing apoptosis in human leukemia cells. Bioscience, biotechnology and biochemistry, 78(10), 1731-1737.

Zare, H. (2019). Effects of Salvia Officinalis Extract on the Breast Cancer Cell Line. SciMedicine Journal, 1(1), 25-29.

Zhang, Z., Wang, X., Zhao, M., \& Qi, H. (2014). Oacetylation of low-molecular-weight polysaccharide from Enteromorpha linza with antioxidant activity. International journal of biological macromolecules, 69, 39-45.

Zhang, Z., Zhang, Q., Wang, J., Song, H., Zhang, H., \& Niu, X. (2010). Chemical modification and influence of function groups on the in vitroantioxidant activities of porphyran from Porphyra haitanensis. Carbohydrate Polymers, 79(2), 290-295.

\section{Abbreviations}

LLPs Polysaccharide extracted from loquat leaves

Ac-LLPs Acetylated LLPs

DS The degree of substitution

FT-IR Fourier-transform infrared spectroscopy

RSM Response surface methodology

ROS Reactive oxygen species

DPPH 1,1-diphenyl-2-picrylhydrazyl

Tris 2-Amino-2-hydroxymethyl-1,3-propanediol

$\mathrm{R}^{2} \quad$ The determination coefficient

$\mathrm{R}^{2}$ adj $\quad$ Adjusted determination coefficient

C.V. The coefficient of the variation

Vc Vitamin C 